\title{
Analysis of the Correlation between the Level of Contact Degradation and the Dynamic Resistance Curve in Circuit Breakers
}

\author{
Ronimack Trajano de Souza and Edson Guedes da Costa \\ Department of Electrical Engineering, Campina Grande Federal University, Campina Grande, 58429-900, Brazil
}

Received: January 23, 2014 / Accepted: February 27, 2014 / Published: June 30, 2014.

\begin{abstract}
The DRM (dynamic contact resistance measurement) in high voltage circuit breakers is a manner of evaluating the internal ageing condition of the chamber. DRM is similar to static contact resistance measurement testing, but instead of measuring a single value when the breaker contacts are closed (static value), the ohmic resistance is measured at various contact positions, from the beginning of the contact opening until a complete separation of the contacts. The relationship between the contact resistances of the new circuit breaker and the ageing circuit breaker in operation provides subsidy for the evaluation of both the main and arcing contact conditions. This research aims to analyze the correlation between the various levels of degradation of the contacts and the configuration of the DRM curve. This work considers curve samples from new breaker chamber contacts and different levels of degradation by acceleration tests.
\end{abstract}

Key words: DRM (dynamic contact resistance measurement), circuit breaker, dynamic resistance, contact resistance, resistance curve.

\section{Introduction}

Circuit breakers are mechanical maneuver devices designed to conduct, restore and interrupt electrical currents. Their main function is to interrupt the defective currents in a given circuit in the shortest possible time, before thermal and mechanical effects can cause harm to people and to the protected electrical installation.

Circuit breakers are classified according to the principle used in extinguishing the electric arcing within its extinction chamber.

The extinction chamber is a compartment of the breaker involving fixed and mobile contacts, and the main contacts and arcing contacts are associated to these [1].

The main contacts are responsible for the load

Corresponding author: Ronimack Trajano de Souza, professor, research fields: circuit breakers, high voltage equipment, electrical maintenance techniques and industrial automation. E-mail: ronimack.souza@ee.ufcg.edu.br. current conduction, and are not submitted to arcing current. Thus, they do not degrade by the effects of the arcing. The arcing contacts are responsible for the conduction of the arcing current, and therefore are submitted to the arcing discharge and all of its effects during the faulty opening operation [2-4].

The energy released by the electric arcing has the ability to induce rapid wear and even the destruction of the contacts if the arcing is not extinguished quickly.

The state of the internal components of the arcing extinguishing chambers in high voltage circuit breakers reflects directly on the working condition of the equipment [2].

The high level of wear of the arcing contacts in circuit breakers directly reflects on a significant decrease in the extinction capability of high voltage during a fault condition in the associated electrical circuit [2]. Thus, there is an increased risk for excess heat within the circuit breaker, with the possibility of reaching higher temperatures than the circuit breaker is 
designed for, thereby deteriorating the insulation and causing abnormal wear on the equipment, and the probability of explosion of the breaker.

Because of this, monitoring the state of the arcing extinguishing chamber is essential. However, in most companies, this monitoring is conducted through periodic supervision of the amount of the static resistance between the contacts, following the reference values provided by the manufacturers. In planning the maintenance of circuit breakers, maintenance crews usually establish parameters to be evaluated periodically, which mostly establish a maximum number of operations, length of service and/or interrupted currents performed by the breaker before promoting a routine of electrical tests to evaluate the breaker [5].

Maintenance techniques must produce accurate data for the correct diagnosis of the circuit breaker status. Disassembly of breakers that have no defects means unnecessary expenses, and may also lead to, or accelerate, the onset of new problems in the equipment during reassembly, and in this case making it susceptible to faulty operation and not ensuring the reliable supply of electricity.

In the analyses of the operating state and the components of the circuit breakers, the arcing extinguishing chambers are one of the main points to be evaluated. Currently, the assessment of the state of the contacts in the arcing extinguishing chambers is made through periodic supervision of the static contact resistance, however, low levels of resistance do not mean chambers in good condition [2-4], considering that the static resistance does not evaluate the state of the main contacts and the arcing contacts separately.

To produce the most enlightening data for measuring contact resistance, research has been directed toward measuring the DRM (dynamic contact resistance measurement) in high voltage circuit breakers as a means of evaluating the internal state of the chambers, by analyzing both the main contacts and the arcing contacts. The curve generated in the DRM allows a more detailed assessment of the state of the main contacts and of arcing contacts, when compared to results obtained from the static contact resistance testing [2-4].

Although DRM is characterized as a promising technique to assess the status of the arcing extinguishing chambers of the circuit breakers, there is still no conclusive model which correlates the dynamic resistance curve to the level of contact wear.

This work aims to evaluate a correlation among the various levels of degradation of contacts, and the configuration of the dynamic contact resistance curve in high voltage circuit breakers. In the development of this research, samples of new fixed and mobile contacts were used to insert distinct levels of degradation derived from accelerated wear testing and samples of fixed and mobile contacts degraded during service. DRM curves were obtained by applying direct test current with an amplitude of $250 \mathrm{~A}$.

\section{Static Contact Resistance}

The measurement of static resistance between the contacts to know the state of connections is a technique used in several decades. Static contact resistance is measured by injecting a DC current through the breaker under test while the breaker is closed and measuring the voltage drop [6-8]. It is recommended that for medium and high voltages the resistance test be made with a microhmmeter having at least 100 A DC output. The use of a higher current value gives more reliable results than using lower current values. The resistance value is usually measured in micro-ohms $(\mu \Omega)$. ANSI C37.09 specifies that the test current should be a minimum of $100 \mathrm{~A}$ [6].

The results have been a decisive factor in assessing the state of the contacts of the arcing extinguishing chambers of medium and high voltage circuit breakers. However, the test does not guarantee that low resistance values imply in good condition arcing contacts, since the measurement is made while the 
breaker is closed, and gives no indication of the condition of the arcing contacts, however, the wear from the arcing current is more focused on the arcing contacts, which are subjected to the heat and energy produced by the arcing during faulty opening operation [2-4]. To evaluate the condition of arcing contacts, an internal inspection may be required, making the breaker unavailable for a long time and demanding high maintenance costs [2-9]. If the internal components of the breaker can be evaluated without the need for disassembly, it is possible to follow the evolution of component wear efficiently, making the maintenance process optimized [9].

In order to obtain more precise data in testing contact resistance in high voltage circuit breakers, researches have been developed to apply DRM, which enables obtaining measurement results which include resistance of the main contacts and arcing contacts in a single electrical testing.

\section{Dynamic Contact Resistance}

The DRM testing is quite similar to static contact resistance measurement testing, but instead of measuring a single value, when the breaker contacts are closed (static value), the resistance is measured under various conditions of connection contacts, from full connection between mobile and fixed contacts, to a complete separation of contacts including measurements at various points of partial connection between mobile and fixed contacts. The DRM results are usually presented in resistance curves $\mathrm{R}(\Omega)$ versus distance contacts $(\mathrm{mm})$ or $\mathrm{R}(\Omega)$ versus opening time(s) of the contacts.

The DRM curve is obtained by applying a high current through the breaker contacts and simultaneous monitoring the voltage drop across the main contact during the operation of the breaker $[2,4,8]$.

DRM testing has been performed by maintenance personnel with the aid of breaker analyzers (switching analyzers), which have a built-in microhmmeter, and the DRM is performed with the injection of a direct test current in the range of $200-250 \mathrm{~A}$, at a nominal speed of contact opening, according to the manufacturer of each model.

Fig. 1 shows a typical assembly scheme used in dynamic contact resistance measurement testing which uses a circuit breaker analyzer. This device has 5 terminals. In voltage sense, 3 banana jacks are used to connect the device under test for recording the voltage drop. Two channels may be connected to a common zero. These signals are transferred to the voltage sense out output connector, which fits neatly to one 3-pole connector, and in current out, two outputs for connecting the current cables to the device under test.

A direct test current, which circulates among the main contacts and the arcing contacts of the breaker, is injected analogically into the static resistance test. Simultaneously, the voltage drop across the contacts (terminal bushings) during a contact opening is measured. Resistance is calculated inside the instrument by direct application of Ohm's law, by dividing the value of the voltage drop across the contacts by the test current injected during all the process of contact opening, resulting in a resistance curve of the main contacts and arcing contacts.

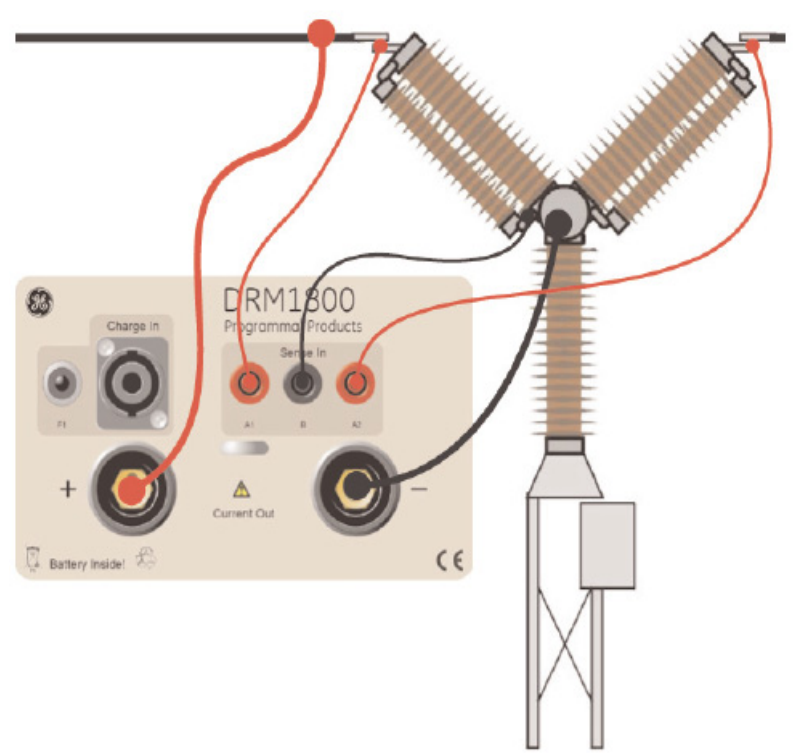

Fig. 1 Assembly scheme of dynamics resistance measurement in circuit breakers (courtesy of GE/Programmer Sweden). 
The study of dynamic resistance provides more conclusive results for circuit breakers that have DRM curves obtained from before installation and service of the circuit breaker (new circuit breaker) and various levels of degradation from the wear of opening/closing operations during service through periodic DRM monitoring [2].

A comparative assessment of the configuration of DRM curves during the life of the circuit breaker will subsidize determining the level of wear of the main contacts and the arcing contacts, contributing to the decision-making of the operating state of the circuit breaker.

The DRM curve can be obtained at the closing or opening of the breaker contacts, in several contact configurations of contacts, however, the dynamic resistance measurements do not provide good results in the closing operation of the circuit breaker, because in that case, the existing direct current, at the moment when there is galvanic connection of the arcing contacts, generates an undesirable noise, impairing the results [4].

Fig. 2 shows an DRM curve during a closing/opening operation obtained in the laboratory for a MOCB (minimum oil circuit breaker)-15 $\mathrm{kV} / 800 \mathrm{~A}$. It is observed that the curve is not symmetrical, and for the curve obtained during the closing of the contacts, peaks of contact resistance are displayed, cited earlier as a consequence of the noise during the measurement.

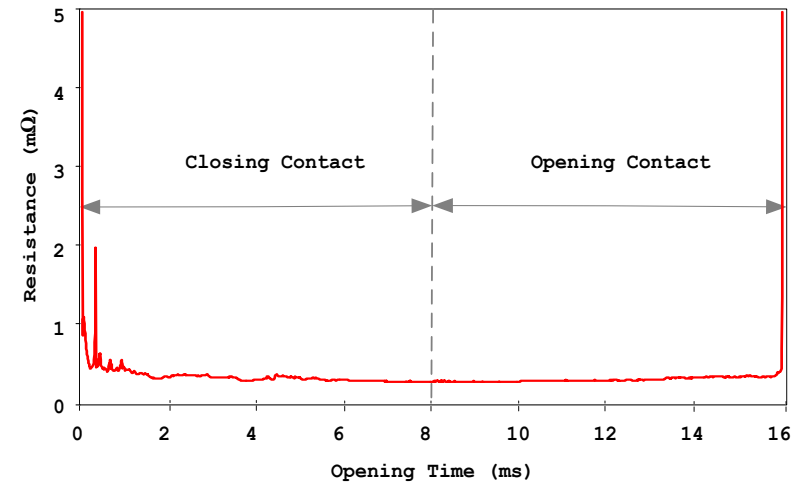

Fig. 2 Closing and opening DRM curve-MOCB 15 kV/800 A.

\section{The DRM Block Diagram and Principle of Operation System}

To perform DRM testing in the circuit breakers, a system which was designed and developed in this research was adopted. This alternative DRM system was developed by using a 4-channel digital oscilloscope, a stationary battery 12 V/220 Ah, resistors produced from Nickel-Chrome wire, current and voltage sensors.

In the test to obtain the DRM curve, the measuring system which was used has the following characteristics:

- capability of direct current injection of up to 300 A (voltage source: $12 \mathrm{~V}$ stationary battery);

- adjustment of the battery current through Nickel-Chrome resistor (40 m $\Omega-1.3 \Omega / 4 \mathrm{~kW}$ );

- measuring of the current with current shunt (500 $\mathrm{A} / 60 \mathrm{mV})$ and voltage/voltage transducer $(60 \mathrm{mV} / 5$ $\mathrm{V})$;

- acquisition of the information captured in the DRM (current and voltage drop across the contacts) using a digital oscilloscope.

Fig. 3 shows the simplified block diagram of the system proposed and adopted in the development of this research.

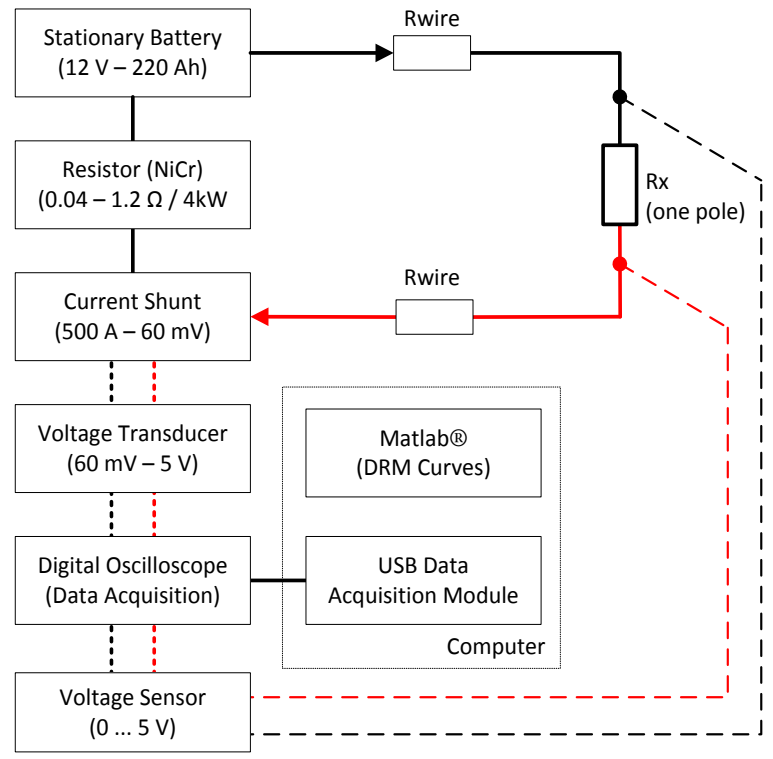

Fig. 3 Block diagram of the DRM system. 
Direct current from the battery between the breaker contacts is applied to measure the DRM curve. A NiCr resistor is inserted in series with the breaker contacts to limit the voltage between the contacts. By adjusting the resistance of the $\mathrm{NiCr}$ resistor, the desired level of current between the breaker contacts is established. The current signal between the breaker contacts will be acquired from a shunt system of a current/voltage transducer $(500 \mathrm{~A} / 5 \mathrm{~V})$ and sent to one of the oscilloscope channels. Simultaneously, the voltage drop signal across the breaker contacts will be acquired by the second channel of the oscilloscope. With the current signals and voltage drop between eligible contacts to be acquired by the oscilloscope, an opening operation of the circuit breaker contacts will be obtained.

Fig. 4 shows a photograph of the arrangement assembled in the laboratory for DRM testing. The test setup that was used to perform DRM at $250 \mathrm{~A}$ DC on a MOCB.

Besides the characteristics of the DRM system presented above, the proposed system enables the achievement of the DRM curve in controlled speed for contact opening. That is the reason for the illustration of an asynchronous motor and frequency inverter in the photograph below.

Fig. 5 shows the flowchart for DRM testing in the proposed system.

The proposed system adopted in this research has been used in laboratory tests. The error appearing in the results depends on the accuracy of current and voltage sensors, since the necessary signals are directly measured and captured by the oscilloscope.

For each stage of contact degradation, current and voltage drop signals were measured and digitized by the oscilloscope. Both signals were entered as input data in the computational routine to calculate the point to point resistance, resulting in the DRM curve.

The analysis of the configuration of the DRM curve will provide subsidy to evaluate the correlation between the curve and the degradation level of the contacts.

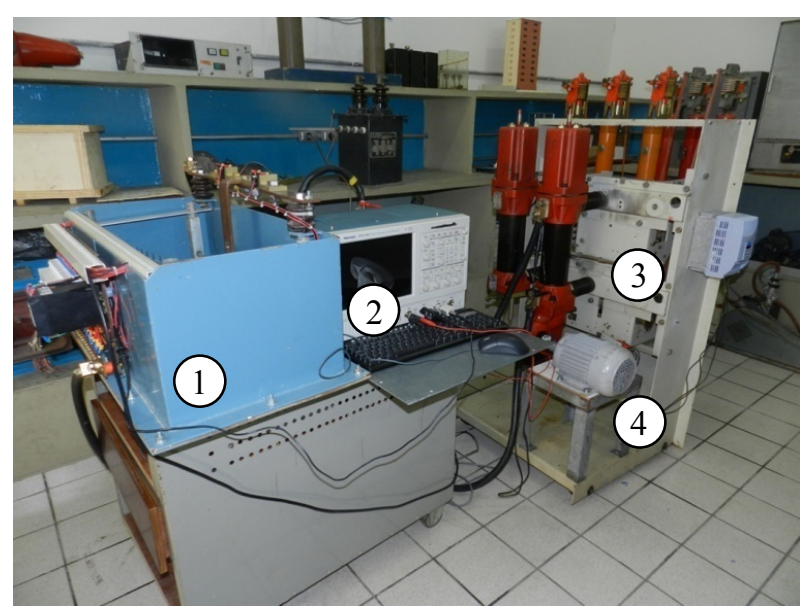

Fig. 4 Test setup for DRM at 250 A DC on a MOCB. 1: Injection system and current measurement; 2: Digital oscilloscope (data acquisition); 3: MOCB; 4: Asynchronous motor.

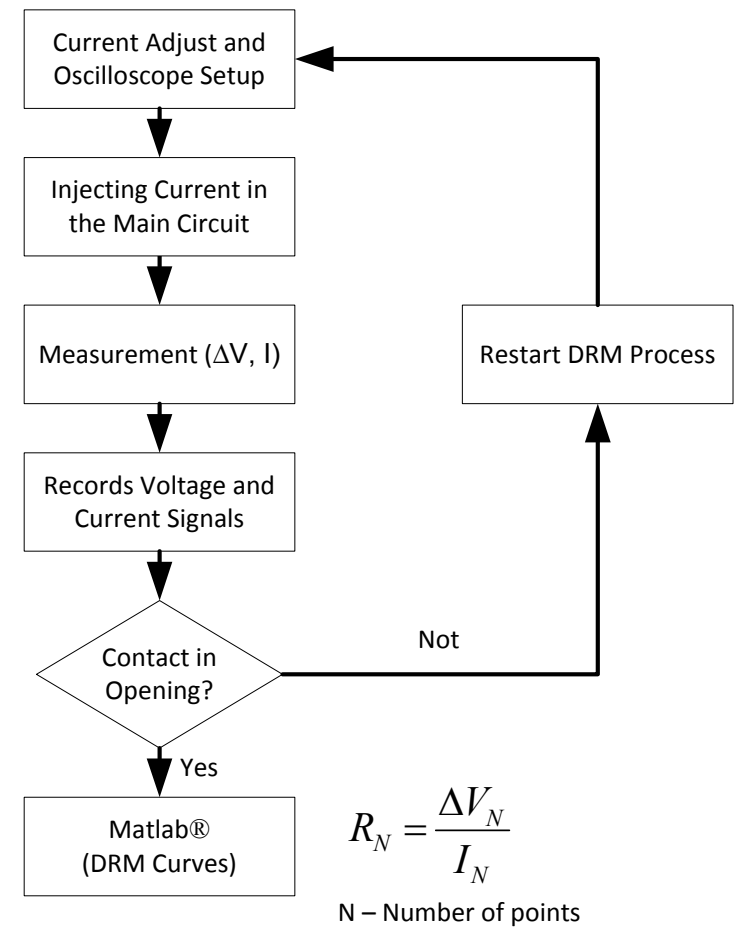

Fig. 5 Flowchart of the DRM test.

\section{Degradation Tests}

To obtain a greater accuracy in the diagnosis of the level of degradation of circuit breaker contacts through the DRM curve, monitoring of the degradation process since the initial stage, new contacts, until the advanced stages of degradation, and degraded contacts due to electric stress (electric arcing and overcurrent) is suggested. 


\section{Dynamic Resistance Curve in Circuit Breakers}

For the development of this research, three pairs of new contacts, called samples (A, B and C) were used. Each sample consists of a fixed contact and a mobile contact with a nominal current of 800 A. In Fig. 6, a photograph of the type of contacts used is presented.

The initial purpose of the degradation tests is to program acceleration of the wear process of the main and arcing contacts by applying short circuits and overcurrent between the contacts.

Before and after each application session in the degradation tests, DRM tests were performed, with contact opening at nominal speed. The objective is to identify, from the DRM curves, which parameters can be monitored to establish a correlation with the level of degradation of the contacts.

The measuring system developed in this research was used to obtain the DRM. Fig. 7 shows photographs of the contacts before and after the degradation.

Fig. 8 shows photographs of the contacts before and after the accelerated degradation in laboratory tests. The fixed and mobile contacts referring to sample "B" were degraded in laboratory, while the fixed and mobile contacts referring to sample " $\mathrm{C}$ " were degraded over the years in which the breaker was in service.

The DRM curves obtained from the contacts degraded in laboratory were confronted with each other, and compared to the DRM curves for the contacts degraded in service. The DRM results are presented in next topic, highlighting the points of galvanic contact between the main and arcing contacts.

\section{Results}

To evaluate the results obtained, the DRM curves were compared at various levels of degradation. In Fig. 9, DRM curves of the new contact are shown in over five different degradation levels for sample "A".

Analyzing the generated curves, it is observed that the DRM curve is not significantly altered in the main contact region, given that the degradation tests caused greater wear on the arcing contact region, as shown in Fig. $7 b$.

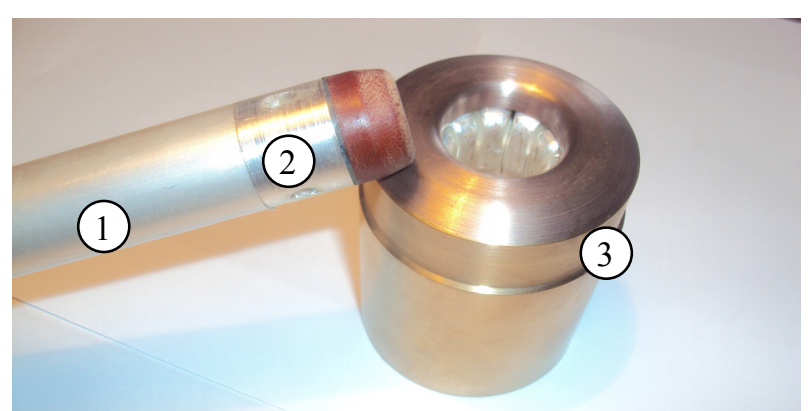

Fig. 6 Type of contacts used for DRM at 250 A DC on a MOCB. 1: Mobile contact (main contact); 2: Mobile contact (arcing contact); 3: Fixed contact (main contact).

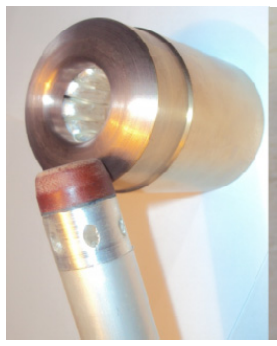

(a)

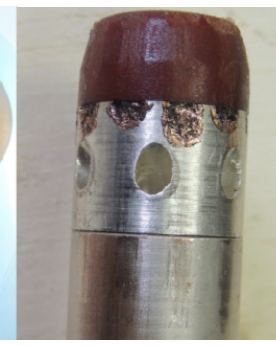

(b)

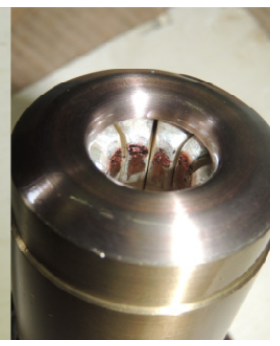

(c)
Fig. 7 State of the contacts after degradation tests on sample "A". (a) New contacts; (b) Mobile contact degraded in laboratory; (c) Fixed contact degraded in laboratory.

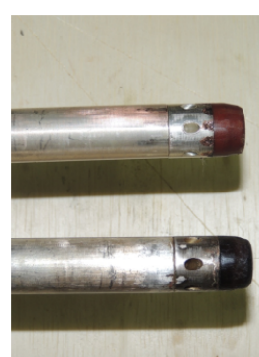

(a)

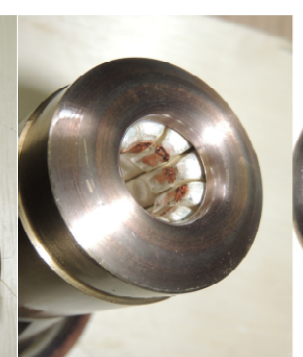

(b)

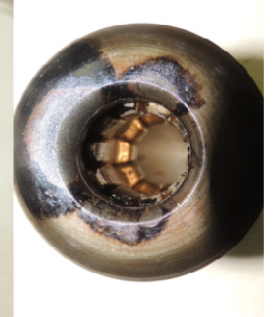

(c)
Fig. 8 State of the contacts after degradation tests. (a) Mobile contact of sample " $B$ "- superior and mobile contact of sample "C"-inferior; (b) Fixed contact of sample "B"; (c) Fixed contact of sample "C".

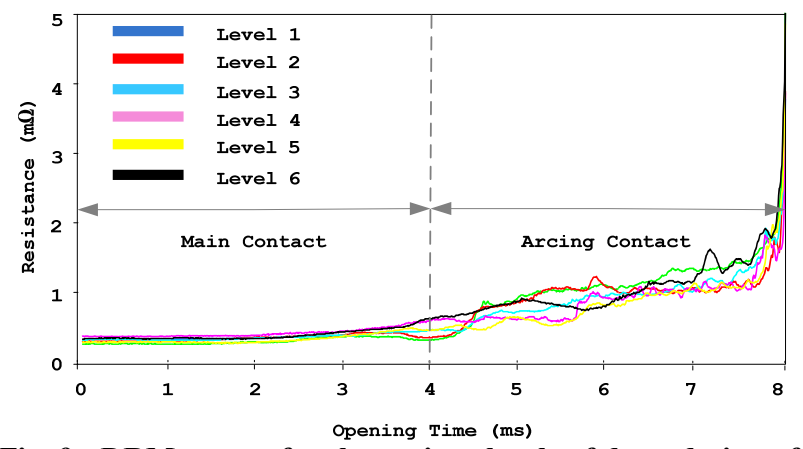

Fig. 9 DRM curves for the various levels of degradation of the contacts on sample "A", obtained at nominal contact opening speed with the application of direct current test of 250 A. 


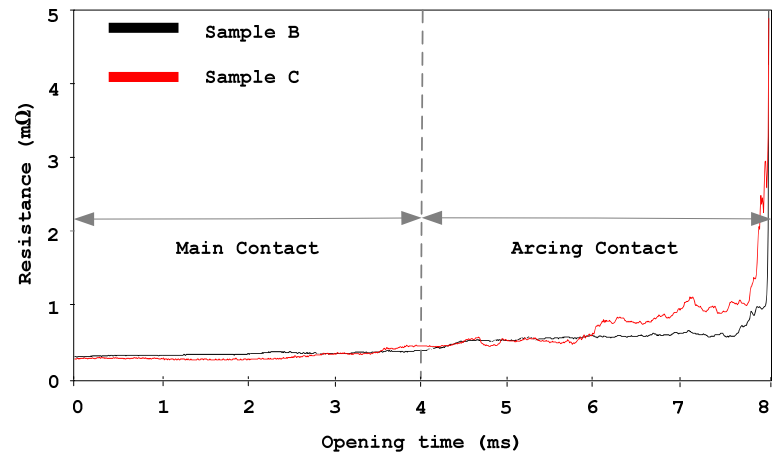

Fig. 10 DRM curves for two levels of degradation of the contacts obtained at nominal contact opening speed with the application of a direct test current of $250 \mathrm{~A}$.

Fig. 10 shows two DRM curves at two different stages of degradation. The black curve represents the DRM at an intermediate stage of degradation for sample "B", while the red curve represents the DRM at a more advanced stage of degradation for sample " $\mathrm{C}$ ". The sample "C" used has more 10 year of use in MOCB.

In Fig. 10, the DRM curve regarding sample "B" shows no significant change in the main contact region. In the region of eminence of complete separation of the contacts (arcing contact), there was a small increase in contact resistance, considering that the degradation tests caused only a little wear on the arcing contact region, as shown in Fig. 8. By analyzing the curve referring to sample "C", it is observed that there is a significant increase in contact resistance, especially on the arcing contact region, with a tendency of evolving into the region of the main contact. The increased resistance is due to the fact that this contact shows the presence of by-products caused by the electric arcing which adhered to the contact during opening operations in failure, in addition to the wear, causing an increase in contact resistance of the circuit breaker.

In both graphs of Figs. 9 and 10, the configuration of the curve changes depending on the level of wear. It is observed that there is an evolution in the value of contact resistance, with increased resistance directly associated to evolution of the level of wear to contacts, with a steeper evolution of resistance in the region of eminence of the total separation of arcing contacts.
The average contact resistance of main contacts for $15 \mathrm{kV}$ class circuit breakers is approximately between 200 and $250 \mu \Omega$ to main contact [6].

\section{Conclusions}

In its current stage, this research has shown relevant data regarding the application of DRM as an efficient parameter in monitoring contacts of circuit breakers.

Based on the results obtained in the experimental trials that were conducted and their analyses, it can be concluded that:

- DRM at nominal speed and an applied current of 250 A to the MOCB, has shown proven reproducibility in the tests for the same contact, both for new contacts and for degraded contacts, which helps to consolidate the DRM at nominal speed.

- Degradation tests provoked wear on the fixed and mobile contacts by applying short circuits and overcurrent between the contacts;

- The wear on the contacts has been identified in the DRM curve markedly in the region of eminence of total separation of contacts (arcing contacts), and were not identified by static resistance testing, which helps to consolidate the use of DRM in the evaluation and diagnosis of the level of degradation of the main and arcing contacts;

- The static resistance testing in this model of MOCB not produced accurate data for the correct diagnosis of contacts degradation;

- The results indicate that the evolution of the DRM curve is directly proportional to the level of wear of the main and arcing contacts, and it becomes more intense in the region of separation of the arcing contacts.

\section{Acknowledgments}

The authors wish to express their thanks to the IFPB (Federal Institute of Paraiba), campus in João Pessoa, for the availability of the first author. The Post-Graduate Program in Electrical Engineering-PPgEE-COPELE, in the Department of Electrical Engineering, Campina Grande Federal 
Dynamic Resistance Curve in Circuit Breakers

University (UFCG) for funding the publish paper. The High Voltage Laboratory of the UFCG and the ATECEL have pump fund into the research.

\section{References}

[1] E. Nasrallah, F. Brikci, S. Perron, Electrical contacts in MV \& HV power circuit breakers, Electric Energy T \& D Magazine 11 (2007) 50-55.

[2] M. Landry, O. Turcotte, F. Brikci, A complete strategy for conducting dynamic contact resistance measurements on HV circuit breaker, IEEE Transactions on Power Delivery 23 (2) (2008) 710-716.

[3] O. Turcotte, R. Gauthier, A thorough examination for circuit breaker health, Transmission and Distribution World 60 (2) (2008) 28-32.

[4] M. Landry, A. Mercier, G. Ouellet, C. Rajotte, J. Caron, M. Roy, et al., A new measurement method of the dynamic contact resistance of HV circuit breakers, in: Proc. 2006 IEEE PES Transmission and Distribution Conference and
Exposition, 2006, pp. 1002-1009.

[5] M. Adam, A. Baraboi, C. Pancu, Monitoring and diagnostic system for high voltage circuit breakers, in: Proc. 2007 International Conference on Electromechanical and Power Systems, 2007, pp. 55-60.

[6] G. Paul, Electrical Power Equipment Maintenance and Testing, CRC Press, Boca Raton, USA, 2008.

[7] J.J. Shea, J.A. Bindas, Measuring molded case circuit breaker resistance, in: Proc. 1992 IEEE Holm Conference on Electrical Contacts, 1992, pp. 159-165.

[8] Z. Stanisic, R. Neimanis, A New ultra lightweight method for static and dynamic resistance measurements, in: Proc. 2010 Conference Record of the IEEE International Symposium on Electrical Insulation (ISEI), 2010, pp. 1-4.

[9] E.G. da Costa, T.A. Felix, A.D. Germano, G.J.C. Almeida, V.C. de Souza, Estudo das características de curvas de resistência dinâmica de disjuntores utilizando redes neurais artificiais, in: Proc. 2010 Simpósio Brasileiro de Sistemas Elétricos (SBSE), 2010. 